\title{
ACTIVIDAD ANTIUROLÍTICA DEL EXTRACTO ETANÓLICO DE PROPÓLEO AYACUCHANO EN RATAS
}

\author{
Rita López-Cabanillas 1,2,a, Ronal Huayanay-Conde ${ }^{1, a}$, Cinthya Gonzales 1,2,a , Mahli Maguiña 1,2,a, \\ Italo Granados ${ }^{1,2, a}$, Mila Ccasani ${ }^{1,2, a}$, Astrid Laguna ${ }^{1, a}$, Ernesto León ${ }^{1, a}$, Nuria Romero-Caballero ${ }^{1, a}$, Jessenia Chacón ${ }^{1, a}$
}

\begin{abstract}
RESUMEN
Objetivos. Determinar la actividad antiurolítica del extracto etanólico de propóleo ayacuchano en un modelo preventivo de urolitiasis inducido en ratas. Materiales y métodos. Se estudiaron 45 ratas albinas macho Sprague-Dawley. El efecto antiurolítico se analizó en cinco grupos de seis animales: blanco, control $(1 \mathrm{~mL}$ de etilenglicol al $5 \%$ y $1 \mathrm{~mL}$ cloruro de amonio al $5 \%$ ) y tres grupos tratados con el extracto etanólico de propóleo a dosis diaria de 250,350 y $500 \mathrm{mg} / \mathrm{Kg}$. Las sustancias fueron administradas mediante canulación orogástrica durante 16 días. El efecto diurético se evaluó en 15 ratas distribuidas en cinco grupos: blanco, control (furosemida $20 \mathrm{mg} / \mathrm{Kg}$ ) y tres grupos tratados con extracto etanólico de propóleo a dosis de 250,350 y $500 \mathrm{mg} / \mathrm{Kg}$. Se midió el pH urinario, densidad urinaria y sedimentación del oxalato de calcio; la presencia de cálculos renales se evaluó mediante cortes histopatológicos por tinción con hematoxilina-eosina bajo luz polarizada. Resultados. El extracto etanólico de propóleo ayacuchano produjo cambios significativos en los valores de ácido úrico, lactato deshidrogenasa sérico, $\mathrm{pH}$, densidad urinaria al comparar los tres grupos dosis; en el análisis histológico observado a luz polarizada se observó menor presencia de cristales de oxalato de calcio en células tubulares del riñón en el grupo tratado a dosis de $250 \mathrm{mg} / \mathrm{Kg}$; el efecto diurético en el grupo tratado con dosis de $250 \mathrm{mg} / \mathrm{kg}$ fue mayor en comparación con la furosemida. Conclusiones. El extracto etanólico de propóleo ayacuchano presenta actividad antiurolítica en el modelo preventivo de urolitiasis en ratas albinas.
\end{abstract}

Palabras clave: urolitiasis, furosemida, propóleo, ratas (Fuente: DeCS BIREME)

\section{ANTIUROLITHIC ACTIVITY OF THE ETHANOLIC EXTRACT OF AYACUCHANO PROPOLIS IN RATS}

\begin{abstract}
Objectives. To determine the antiurolytic activity of the ethanolic extract of Ayacuchan propolis in a preventive model of urolithiasis in rats. Materials and methods. A total of $\mathbf{4 5}$ male Sprague-Dawley albino rats were studied. The antiurolithic effect was analyzed in five groups of six animals each: blank, control (treated with $1 \mathrm{~mL}$ of $5 \%$ ethylene glycol and $1 \mathrm{~mL}$ of $5 \%$ ammonium chloride), and three experimental groups (treated with the ethanol extract of propolis at a daily dose of 250,350 , and $500 \mathrm{mg} / \mathrm{kg}$, respectively). The drugs were administered by orogastric cannulation for 16 days. The diuretic effect was evaluated in 15 rats distributed in five groups: blank, control (treated with furosemide at $20 \mathrm{mg} / \mathrm{kg}$ ), and three experimental groups (treated with the ethanol extract of propolis at daily doses of 250,350 , and $500 \mathrm{mg} / \mathrm{kg}$, respectively). Urinary $\mathrm{pH}$, urinary density, and sedimentation of calcium oxalate were measured. The presence of kidney stones was evaluated by examination of hematoxylin-eosin-stained histopathological sections under polarized light. Results. The ethanolic extract of Ayacuchan propolis caused significant changes in the levels of uric acid, serum lactate dehydrogenase, $\mathrm{pH}$, and urinary density in the three dose groups. The results of histological analysis indicated a lower presence of calcium oxalate crystals in kidney tubular cells in the group treated with $250 \mathrm{mg} / \mathrm{kg}$. The diuretic effect in the group treated with $250 \mathrm{mg} / \mathrm{kg}$ was higher than that in the control group. Conclusions. The ethanolic extract of Ayacuchan propolis demonstrated antiurolytic activity in a preventive rat model of urolithiasis.
\end{abstract}

Key words: urolithiasis, furosemide, propolis, rats (source: MeSH NLM)

\section{INTRODUCCIÓN}

Según la Organización Mundial de la Salud (OMS), la urolitiasis es la tercera causa de enfermedades más frecuente en las vías urinarias. En el Perú, cerca del $10 \%$ de hombres y el $5 \%$ de mujeres se verán afectados por esta enfermedad a lo largo de su vida. El desarrollo de los cálculos renales está asociado a una alta tasa de recidiva dependiente del tiempo en los pacientes tratados, estimándose en un $10 \%$ al año y hasta un $50 \%$ a los 10 años ${ }^{(1)}$.

\footnotetext{
Facultad de Medicina, Universidad Nacional Mayor de San Marcos. Lima, Perú.

Sociedad Científica de San Fernando. Facultad de Medicina, Universidad Nacional Mayor de San Marcos. Lima, Perú.

Estudiante de Medicina.

Recibido: 06/03/2017 Aprobado: 08/11/2017 En línea: 06/12/2017
}

Citar como: López-Cabanillas R, Huayanay-Conde R, Gonzales C, Maguiña M, Granados I, Ccasan M, et al. Actividad antiurolítica del extracto etanólico de propóleo ayacuchano en ratas. Rev Peru Med Exp Salud Publica. 2017;34(4):642-8. doi: 10.17843/rpmesp.2017.344.2730 
La urolitiasis consiste en el depósito de cristales de diferentes compuestos, en el $80 \%$ de los casos es oxalato de calcio cuya formación depende de diversas condiciones del medio como la presencia de hiperoxaluria y alteraciones del $\mathrm{pH}$ urinario ${ }^{(2)}$. El inicio de la formación de cristales de oxalato es la nucleación, la cual ocurre por la sobresaturación de la orina. Estos cristales dañan a las células de los túbulos renales y ocasionan una pérdida de la polaridad celular ${ }^{(3,4)}$.

El etilenglicol (EG) es un precursor metabólico del oxalato, por lo que su administración a animales de experimentación resulta en hiperoxaluria y cristaluria al inducir efectos modificantes en la orina como sobresaturación, inactivación de los inhibidores naturales de cálculos y disminución del $\mathrm{pH}$ que favorecen el depósito de cristales de oxalato cálcico monohidrato en células tubulares renales ${ }^{(5)}$. La administración de cloruro de amonio (CA), sustancia que promueve la acidosis metabólica, se ha empleado con frecuencia junto al EG para inducir una alta tasa de depósitos uniformes de cristales de oxalato de calcio en modelos experimentales de urolitiasis ${ }^{(6)}$.

El propóleo es una resina producida por las abejas y ha sido reconocido en el tratamiento de diversas enfermedades tanto in vivo como in vitro ${ }^{(7)}$. Un estudio realizado en Perú determinó que los flavonoides, triterpenos y polifenoles son los compuestos mayoritarios del propóleo, principalmente en el de origen ayacuchano ${ }^{(8,9)}$. Los flavonoides, son antioxidantes que han demostrado efectos antibacterianos, antiinflamatorios, antifúngicos, entre otros ${ }^{(10)}$. El lupeol es el triterpeno pentacíclico más abundante identificado en el propóleo y posee efectos contra la inflamación, trastornos renales, entre otros ${ }^{(11,12)}$.

Diversos estudios han revelado la capacidad antiurolítica del lupeol en modelos experimentales inducidos con EG ${ }^{(13,14)}$. Cuando hay daño renal se inhiben los complejos que evitan la formación de cálculos renales tales como pirofosfato, fosfato ácido y citrato, principalmente. El lupeol actúa evitando la inhibición de estos elementos, aumentando la diuresis, inhibiendo la síntesis de oxalato cálcico monohidratado e incrementando la actividad antioxidante ${ }^{(15)}$.

Se denomina actividad antiurolítica a la capacidad de un compuesto de inhibir la nucleación, agregación y depósito de cálculos renales junto a una adecuada función renal evidenciada en la excreción normal de compuestos que predisponen a la formación de cálculos ${ }^{(16)}$.

El procedimiento quirúrgico contra la urolitiasis es la litotricia extracorpórea con ondas de choque (LEOC), sin embargo, puede tener complicaciones como cólicos nefríticos y daño glomerular, así como recurrencia de cálculos renales. A pesar de que algunos medicamentos se han utilizado para prevenir esta recurrencia, aún su eficacia no ha sido demostrada. Ante esto, los estudios sobre el consumo de diversos productos naturales con efectos antioxidantes,

\section{MENSAJES CLAVE}

Motivación para realizar el estudio. La urolitiasis es una enfermedad altamente recidivante, por ello, es necesario optar por alternativas que coadyuven a mejorar la calidad de vida de los pacientes El propóleo, resina natural, es usado como medicina alternativa en diversas patologías. Por ello se necesitan estudios para generar evidencia que avalen su eficacia.

Los principales hallazgos. El consumo de extracto etanólico de propóleo ayacuchano presenta actividad antiurolítica, producto de sus propiedades antioxidantes, en un modelo preventivo de urolitiasis en ratas albinas.

Implicancias. Promover mediante el uso de un producto natural como el propóleo, una alternativa coadyuvante y accesible en el tratamiento de la urolitiasis para la comunidad.

diuréticos y antiurolíticos han demostrado una mayor eficacia, seguridad y adherencia terapéutica para la prevención de la recurrencia de urolitiasis ${ }^{(16)}$.

El presente estudio tiene como objetivo determinar si el extracto etanólico del propóleo ayacuchano, un producto natural de la sierra en Perú, tiene efecto antiurolítico al reducir depósitos de cristales de oxalato cálcico monohidrato y brindar adecuada función renal (efecto diurético) en un modelo experimental de urolitiasis inducida por etilenglicol en ratas.

\section{MATERIALES Y MÉTODOS}

Se realizó un estudio experimental en 45 ratas albinas macho cepa Sprague-Dawley, de 8 semanas de edad, con peso promedio $200 \pm 50 \mathrm{~g}$, procedentes del bioterio de la Universidad Peruana Cayetano Heredia y acondicionadas en el bioterio de la Facultad de Medicina de la Universidad Nacional Mayor de San Marcos por siete días previos, con agua y alimento ad libitum, ciclo luz-oscuridad de $12 \mathrm{~h}$ y temperatura de $22-24{ }^{\circ} \mathrm{C}$.

\section{PREPARACIÓN DEL EXTRACTO}

El propóleo artesanal obtenido proviene del centro de producción del valle de Ayacucho, en el departamento de Ayacucho, la entidad proveedora fue la empresa Herbamiel, certificada y aprobada por La Dirección General de Salud Ambiental (DIGESA) y Dirección General de Medicamentos, Insumos y Drogas (DIGEMID). El extracto de propóleo se preparó añadiendo $40 \mathrm{~g}$ de propóleo finamente fraccionado a $100 \mathrm{~mL}$ de etanol al $80 \%$. Se dejó en maceración a temperatura ambiente y protegido de la luz en frasco ámbar durante 7 días. Luego, se decantó el sobrenadante y se concentró en baño maría a $50{ }^{\circ} \mathrm{C}$ por $4 \mathrm{~h}$ a fin de evaporar el etanol hasta obtener una consistencia pastosa. Para administrar la dosis del extracto a los animales de experimentación, este se combinó con propilenglicol utilizado para disolver el propóleo de consistencia resinosa ${ }^{(17-19)}$. 
El extracto de propóleo ayacuchano se sometió a pruebas de espectrofotometría ultravioleta visible (UV-Vis) utilizando un rango de longitud de onda entre 200 hasta $500 \mathrm{~nm}$.

\section{EVALUACIÓN DEL EFECTO DIURÉTICO}

Se evaluó siguiendo la técnica descrita por Leipschitz et al. (21). Los animales se mantuvieron bajo privación de agua y comida las $18 \mathrm{~h}$ previas al inicio del experimento y durante las $6 \mathrm{~h}$ de recolección de la orina. Se emplearon 15 ratas que fueron distribuidas al azar en cinco jaulas de diuresis de tres animales cada una. El grupo Blanco recibió suero fisiológico, el grupo Control recibió furosemida (20 $\mathrm{mg} / \mathrm{kg}$ ), dosis estándar recomendada, disuelta en suero fisiológico; los tres grupos tratados recibieron el extracto a dosis de 250, 350 y $500 \mathrm{mg} / \mathrm{kg}$, respectivamente. Todas las sustancias a dosis de $10 \mathrm{~mL} / \mathrm{kg}$ de peso corporal del animal, fueron administradas por vía oral mediante cánula orogástrica una vez y se colocaron a los animales en las jaulas metabólicas. Se recolectó orina durante seis horas, midiéndose el volumen de excreción urinaria (UVE) obtenido por hora por método convencional (tubo calibrado en $\mathrm{mL}$ ). Para el análisis de las seis mediciones se utilizó la siguiente fórmula: UVE = (volumen recolectado/volumen administrado) $\times 100$.

\section{EVALUACIÓN DEL EFECTO ANTIUROLÍTICO}

Bajo el modelo preventivo de la enfermedad, se indujo urolitiasis administrando solución de etilenglicol 5\% y cloruro de amonio $5 \%$, cuyas proporciones se determinaron en función a los resultados de la prueba piloto y estudios previos ${ }^{(22,23)}$.

Se emplearon 30 ratas albinas macho distribuidas aleatoriamente en cinco grupos de seis animales cada uno. La administración del extracto de propóleo ayacuchano se realizó $6 \mathrm{~h}$ previas a la inducción de la urolitiasis. Todas las sustancias fueron administradas mediante canulación orogástrica durante 16 días, según el siguiente diseño experimental:

Grupo Blanco (B): suero fisiológico $(2 \mathrm{~mL})$; grupo Control (C): $2 \mathrm{~mL}$ de EG $5 \%$ + CA $5 \%$ mezclados en proporciones iguales (solución de inductor); grupo Dosis 1 (D1): $2 \mathrm{~mL}$ de solución de inductor y extracto a $250 \mathrm{mg} / \mathrm{kg}$; grupo Dosis 2 (D2): $2 \mathrm{~mL}$ de solución de inductor y extracto a $350 \mathrm{mg} / \mathrm{kg}$; grupo Dosis 3 (D3): $2 \mathrm{~mL}$ de solución de inductor y extracto a $500 \mathrm{mg} / \mathrm{kg}$.

Se utilizaron jaulas metabólicas con base cubierta con doble malla filtro para la recolección de orina durante 24 $\mathrm{h}$ en ayunas, tanto para la medición basal como después del último día de inducción. Se analizó los niveles de pH urinario, densidad urinaria y sedimentación de oxalato de calcio obtenidos por muestra.

Para la obtención de la muestra sanguínea, se anestesió a los animales y se obtuvo aproximadamente $5 \mathrm{~mL}$ de sangre mediante punción cardiaca, para después centrifugar y obtener el suero. Se obtuvo los niveles de creatinina, ácido úrico y lactato deshidrogenasa (LDH) por muestra. Posterior a ello, se sacrificó a los animales para extraerles los riñones. Estos se lavaron con solución salina para limpiar la sangre y otras adherencias. Se los fijó en solución de formol al 10\% por tres días y se tomó muestras para obtener láminas que fueron teñidas con hematoxilina-eosina $(\mathrm{H}-\mathrm{E})$. Se realizó la medición del tamaño de los cálculos renales encontrados en cada grupo mediante el uso del programa JMicrovision 1.27.

\section{CONSIDERACIONES ÉTICAS}

En el presente estudio el uso y manejo de los animales de experimentación se realizó de acuerdo a Guide for the Care and Use of Laboratory Animals, 8th edition, al Manual de Procedimientos para el uso de animales de laboratorio del Instituto Nacional de Salud y la eutanasia acorde al AVMA (Guidelines for the Euthanasia of Animals: 2013 Edition). El proyecto fue aprobado por el Comité de Ética en Investigación de la Facultad de Medicina de la Universidad Nacional Mayor de San Marcos.

\section{RESULTADOS}

Se observan picos de absorbancia analizados en la espectrofotometría ultravioleta visible del extracto etanólico de propóleo ayacuchano, principalmente un pico correspondiente a la longitud de onda de $295 \mathrm{~nm}$, evidenciando la presencia de flavonoides y triterpenos (Figura 1).

\section{EFECTO ANTIUROLÍTICO}

En la Tabla 1 se muestran los resultados séricos y urinarios encontrados después de la inducción con EG y CA. Se

Tabla 1. Evaluación bioquímica del propóleo ayacuchano posinducción con etilenglicol y cloruro de amonio en un modelo preventivo experimental de urolitiasis en ratas

\begin{tabular}{|c|c|c|c|c|c|}
\hline Variables* & (B) Blanco & (C) Control & (D1) Dosis 1 & (D2) Dosis 2 & (D3) Dosis 3 \\
\hline Ácido úrico (mg/dL) & $0,35 \pm 0,49$ & $0,72 \pm 0,77$ & $0,39 \pm 0,10^{* *}$ & $0,51 \pm 0,35^{* *}$ & $0,81 \pm 0,18^{* *}$ \\
\hline Creatinina (mg/dL) & $0,77 \pm 0,21$ & 0,83 & $0,97 \pm 0,14$ & $0,98 \pm 0,12$ & $1,07 \pm 0,56$ \\
\hline Lactato deshidrogenasa (UI) & $331,0 \pm 41,0$ & $1088 \pm 189,5$ & $704 \pm 189,5^{\star *}$ & $567,5 \pm 166,1^{* *}$ & $893,5 \pm 157,6^{* *}$ \\
\hline $\mathrm{pH}$ & $6,5 \pm 0,7$ & $6,5 \pm 0,7$ & $6,5 \pm 0,7^{* *}$ & $6 \pm 1,4^{\star *}$ & $7,5 \pm 2,1^{* *}$ \\
\hline Densidad urinaria & $1007,5 \pm 3,5$ & $1015 \pm 14,1$ & $1007 \pm 3,5^{\star \star}$ & $1022,5 \pm 3,5^{\star \star}$ & $1007,5 \pm 3,5^{\star \star}$ \\
\hline
\end{tabular}




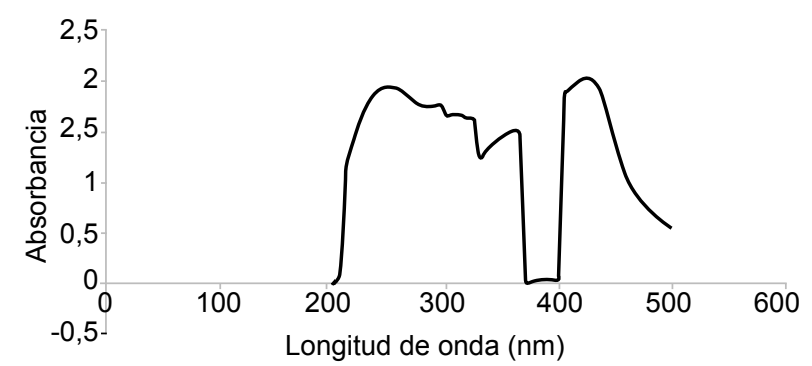

Figura 1. Espectrofotometría ultravioleta visible del extracto de propóleo ayacuchano.

aprecia que el grupo Control presentó un aumento de las cantidades de ácido úrico, creatinina y LDH séricos en comparación con el grupo Blanco. Sin embargo, los valores de creatinina en los grupos Dosis fueron mayores en comparación al grupo Control. En la orina del grupo Control y el grupo Blanco, los niveles del $\mathrm{pH}$ se mantuvieron en el mismo rango. El tratamiento con el extracto de propóleo ayacuchano produjo cambios significativos $(p=0,03$, ANOVA) en los valores de ácido úrico, $\mathrm{LDH}$ séricos, $\mathrm{pH}$, densidad urinaria y en menor cuantía en creatinina al comparar los tres grupos Dosis.
En el análisis radiológico no se evidenció la presencia de figuras radiopacas en las vías urinarias de los grupos Dosis 1,2 y 3 . En el grupo Control se pudo observar la presencia de figuras litiásicas presentes en las vías urinarias.

Para el primer caso se pudo observar que el grupo Blanco mostró una citoarquitectura normal. Por otro lado, el grupo Control presentó extensos depósitos de cristales de oxalato de calcio en los túbulos renales, además de lesiones en dicha estructura como dilatación tubular y edema. De los grupos tratados, el grupo Dosis 1 fue el único que evidenció disminución del tamaño de los depósitos de cristales y menor grado de lesión renal. Además, no se evidenció dilatación tubular ni edema. En el grupo Dosis 3, las muestras presentaron un incremento considerable en el número de depósitos y tamaño de los cristales de oxalato de calcio en comparación con el grupo Control.

Mediante la observación de las láminas histopatológicas a luz polarizada se confirmaron los depósitos de cristales de oxalato de calcio en la luz tubular; estos cristales tienden a ser regulares, laminados o en abanico, son incoloros a tinciones normales y birrefringentes a la luz polarizada. La formación de los cristales estuvo presente en todas las áreas del riñón, incluyendo corteza y médula (Figura 2).
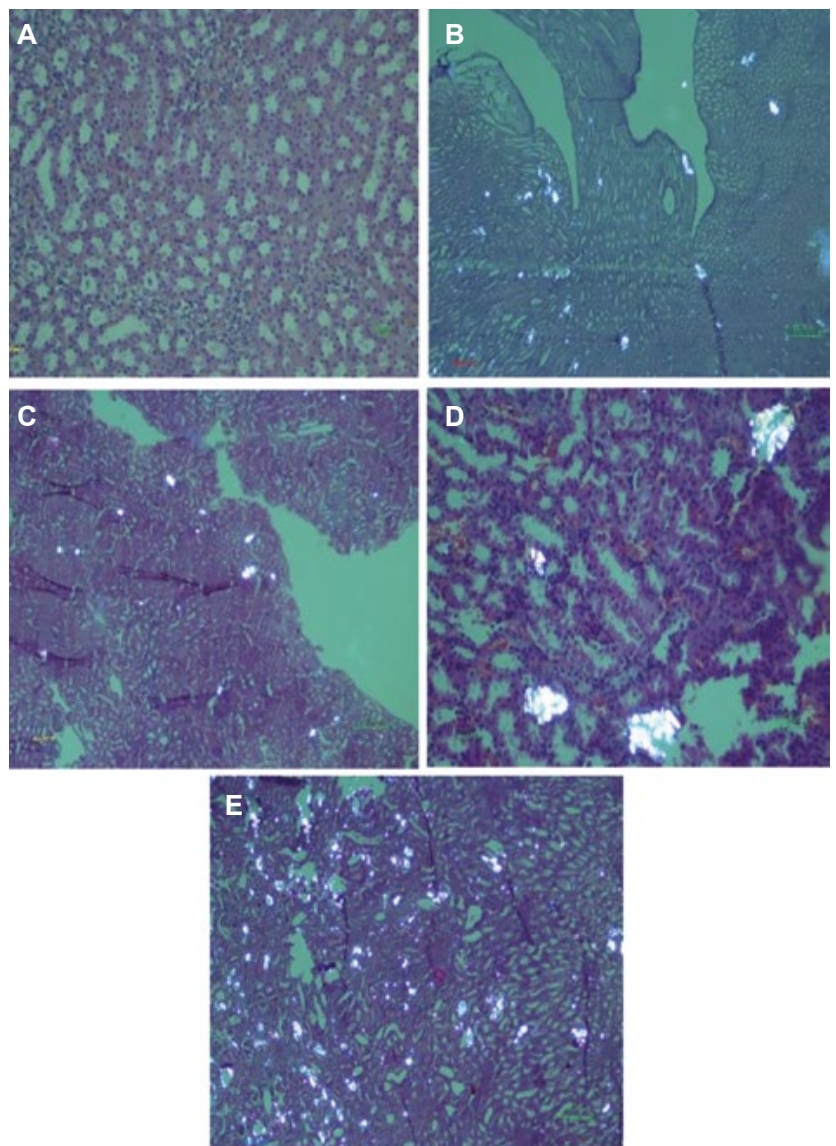

Figura 2. Resultados histológicos observados a luz polarizada (HE) a 20X de los grupos de ratas A) Blanco; B) Control; C) Dosis 1; D) Dosis 2; E) Dosis 3 


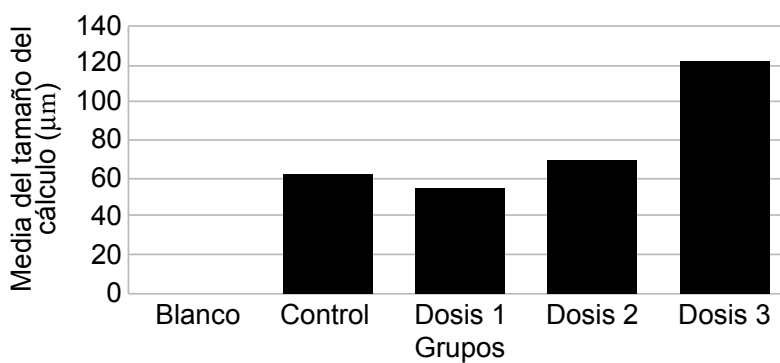

Figura 3. Media de tamaño de los cálculos renales medidos por grupos de ratas

Los tamaños de los cálculos renales encontrados en cada grupo presentaron diferencias estadísticamente significativas $(p=0,01)$. En el grupo Dosis 1 , la media del tamaño de los cristales fue de $54,8 \mu \mathrm{m}$, estos fueron de menor tamaño en comparación al grupo Control, de 61,7 $\mu \mathrm{m}$. Se observó un incremento de tamaño de los cálculos tanto en el grupo Dosis 2 con $67,47 \mu \mathrm{m}$ y Dosis 3 con $121,22 \mu \mathrm{m}$ (Figura 3).

\section{EFECTO DIURÉTICO}

En la evaluación diurética se observa que el volumen es más alto para el grupo Dosis 1, seguido por los grupos Dosis 2 y Dosis 3 , cuyos volúmenes totales fueron iguales y después por el grupo Control y grupo Blanco respectivamente. Se obtuvo diferencias entre los cinco grupos, pero en las primeras $5 \mathrm{~h}$ fue más alto el volumen de orina en los grupos experimentales, siendo el grupo Dosis 1 el que presentó mayor intensidad en la segunda y quinta hora, lo que muestra un efecto diurético mayor de los grupos experimentales que el de la furosemida siendo los resultados significativos para los volúmenes totales $(p=0,021)$ (Figura 4).

En la figura 5, se indica la UVE de todos los grupos, los valores totales tuvieron diferencias significativas $(p=0,003$; ANOVA). Al igual que resultados anteriores, el grupo Dosis 1

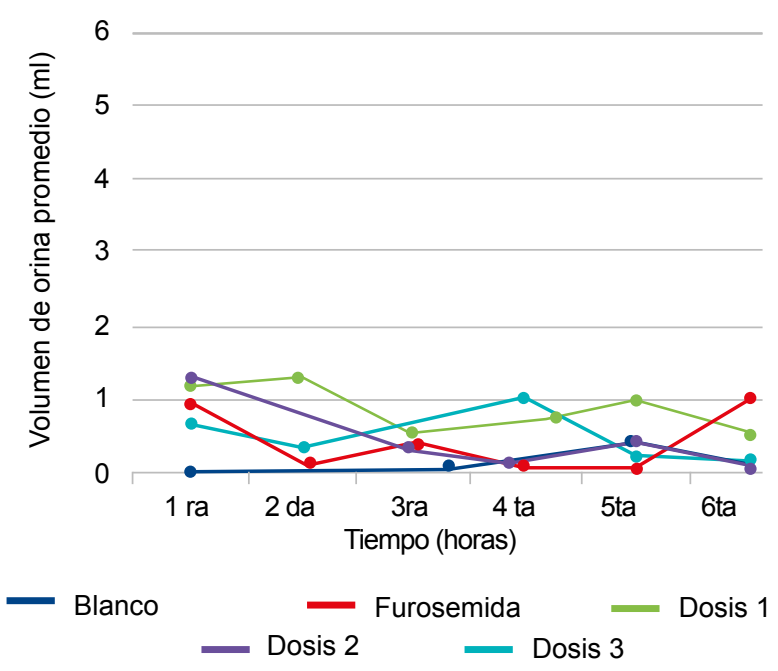

Figura 4. Volumen de orina en seis horas por grupo de ratas

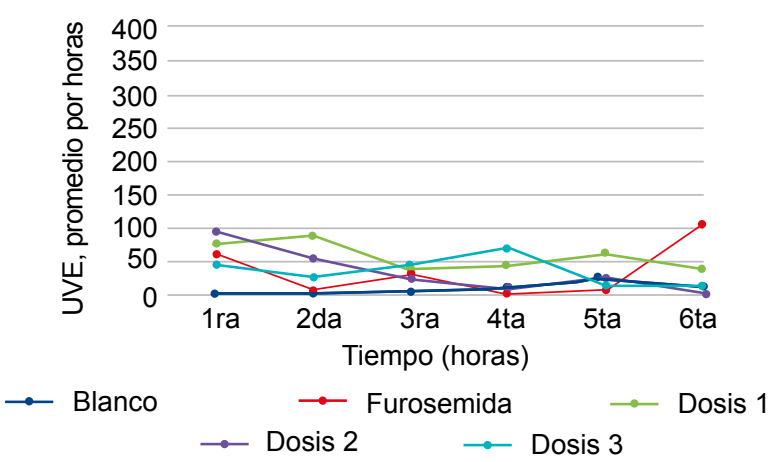

UVE: Volumen de excreción urinaria

Figura 5. Volumen de excreción urinaria calculada en 6 horas por grupos de ratas

presentó la más alta excreción volumétrica de orina, seguido de los grupos Dosis 2 y Dosis 3 y el grupo Control, cuyos valores fueron muy similares. Según la prueba de Bonferroni, se encontró diferencias significativas para las siguientes comparaciones: a la sexta hora, grupo Blanco vs. grupo Furosemida $(p=0,001)$, grupo Furosemida vs grupo Dosis 1 $(p=0,003)$, grupo Furosemida vs. grupo Dosis $3(p=0,001)$.

\section{DISCUSIÓN}

Los cálculos de cristales de oxalato cálcico monohidratado inducidos por el EG generan estrés oxidativo en las células tubulares y, a la vez, daño celular, propiciando un ambiente favorable para su desarrollo. En la urolitiasis, el daño tubular producido por los derivados del EG, principalmente el oxalato, conlleva a una disminución de la reabsorción y secreción tubular fisiológica, en el caso del primero, incrementa la sobresaturación del medio, con mayor formación de cálculos que obstruyen las vías urinarias; por su parte, la disminución de la secreción tubular de sustancias incrementa sus concentraciones séricas también. Ambos efectos, globalmente, llevan a un incremento de metabolitos en sangre, la filtración glomerular disminuye debido a los cálculos que se forman en el sistema urinario que obstruye el flujo y excreción normal de orina ${ }^{(24)}$.

En la evaluación antiurolítica del modelo experimental de urolitiasis, la mayor presencia de sedimento de oxalato cálcico monohidratado fue encontrada en el grupo Dosis 1 , esto podría deberse al desprendiendo de los cálculos con la mucina de los túbulos renales, lo que disminuiría su presencia en el tejido renal debido a su expulsión progresiva como sedimento urinario. Este mecanismo es concluido por Hosseinzadeh et al. (25), fundamentando que, si se inhibe la agregación de los cálculos de oxalato, en consecuencia, se facilita su excreción por la orina. Sin embargo, existe otro estudio que encuentra resultados contrarios a lo postulado ${ }^{(26)}$.

Asimismo, los grupos experimentales, principalmente el grupo Dosis 1, evidenciaron una menor densidad urinaria, en comparación con los mayores valores del grupo Control. El elevado valor para el pH urinario en el grupo Dosis 3 con 
respecto al grupo Control, se condice con otros trabajos realizados por Vyas et al. y Kachchhi et al. ${ }^{(26,27)}$. El EG perturba el metabolismo normal del oxalato al incrementar la disponibilidad de sustrato, de modo que aumenta la actividad de enzimas para la síntesis de oxalato, y la LDH es la enzima principal que cataliza esta formación ${ }^{(28)}$. En los resultados séricos aumentó significativamente la actividad de LDH en el grupo Control, lo cual confirma una acción directa con la deposición de oxalato cálcico monohidratado en este grupo. Los niveles de ácido úrico sérico en los grupos experimentales también se encontraron disminuidos respecto al grupo Control; caso contrario sucede con el nivel de creatinina sérica. Salvo este último hallazgo, los valores obtenidos de estos marcadores de lesión de tejido renal son referidos también en los trabajos de Ghelani et al., Kachchhi et al., Anbu et al. ${ }^{(27-29)}$.

Las siluetas radiopacas observadas se pueden relacionar con posibles figuras litiásicas. La observación de estas figuras está ligada a la velocidad de eliminación de las mismas, así como al tamaño de estas. Si los cálculos formados son muy pequeños, estos se eliminan con facilidad y no son observables a los rayos $\mathrm{X}$. Las placas radiológicas tomadas en la medición final evidenciaron la presencia de posibles cálculos renales formados en las vías urinarias del grupo Control, a comparación de los grupos experimentales en los que no se evidenció ninguna figura litiásica ${ }^{(30)}$.

En cuanto al análisis de los cortes histopatológicos observados en el microscopio de luz polarizada, se deduce que la presencia de cálculos renales en las láminas de los grupos experimentales ha ido aumentando en función al aumento de cantidad de extracto etanólico de propóleo administrado, así es como en el grupo Dosis 3 (500 mg/kg), la excesiva concentración no genera el resultado esperado en la disminución del tamaño de los cristales.

Dado el presente análisis fitoquímico, diversos estudios realizados han encontrado picos de absorbancia que coinciden con el rango de longitud de onda de compuestos flavonoides, que según los hallazgos de Righi et al. (31), Martínez et al. ${ }^{(32)}$, se corresponden con los picos de absorbancia más altos obtenidos en nuestro estudio, por lo que es muy probable que nuestro extracto de propóleo ayacuchano contenga en su composición flavonoides.

En la evaluación del efecto diurético, se postula que el extracto etanólico de propóleo a dosis de $250 \mathrm{mg} /$ $\mathrm{kg}$ provoca mayor diuresis que la furosemida, lo cual es notorio en las primeras $5 \mathrm{~h}$. Este efecto diurético puede ser atribuido a la presencia de metabolitos secundarios como los flavonoides, dada su alta concentración en nuestro extracto, pudo haber ejercido una acción vasodilatadora y osmótica y favorecer el aumento de la diuresis, según lo mencionan otros autores ${ }^{(20)}$. Sin embargo, el mecanismo que interviene en el posible efecto diurético del propóleo debería ser esclarecido en futuras investigaciones.
El probable efecto de inhibición de la formación de los cálculos renales encontrado en el grupo Dosis 1 respecto a los otros grupos, también se correlaciona con los marcadores bioquímicos evaluados, así como en las muestras histopatológicas analizadas, por lo que puede ser considerada la dosis óptima para las condiciones del estudio. Es necesario indicar también que no podemos establecer alguna relación específica entre la actividad antiurolítica y la dosis de propóleo empleado, dado la escasez de estudios que permitan mayor precisión en la composición y efectos del propóleo ayacuchano; por lo que es posible encontrar alguna toxicidad en dosis mayores a $300 \mathrm{mg} / \mathrm{kg}$, siendo muchas veces menor a la dosis tóxica establecida por otros estudios ${ }^{(33)}$.

El presente estudio debe reconocer sus limitaciones; este trabajo plantea la actividad del lupeol como un compuesto antioxidante que desarrollaría un efecto antiurolítico. Si bien el análisis de espectrofotometría ultravioleta visible realizado no demuestra ninguna absorbancia sobre el lupeol, es necesario mencionar que mediante el uso de técnicas más especializadas como HPLC (High Performance Liquid Chromatography), se ha evidenciado y cuantificado el lupeol en extractos de propóleo, por lo que se recomienda el empleo de estas técnicas ${ }^{(27)}$, a la que no pudimos acceder por resultar altamente costosa. También hubiera sido importante evaluar el perfil urinario de metabolitos como el sodio y potasio, y así complementar lo recomendado en diferentes protocolos de evaluación diurética. Es prudente mencionar que esta última no afecta la validez de los resultados para los propósitos del estudio presente ${ }^{(34)}$.

Se concluye que el extracto etanólico de propóleo ayacuchano tuvo una actividad antiurolítica favorecido por sus propiedades antioxidantes y evidenciado en la disminución de los depósitos de cálculos renales y aumento de la diuresis en las ratas tratadas a la dosis de $250 \mathrm{mg} / \mathrm{kg}$. Posteriores estudios deberían cuantificar el lupeol con precisión en el propóleo. Asimismo, se podrían iniciar estudios sobre un probable efecto litotrípsico del propóleo.

Agradecimientos: a la Dra. Silvia Suárez Cunza por la realización del análisis fitoquímico del extracto etanólico al Dr. Diego Díaz Coahila por la obtención de las placas radiográficas; al Dr. Ernesto Nava Carrión por la validación de las muestras histopatológicas realizadas; al Dr. Víctor Mendoza Conco por la validación de los resultados de las placas radiográficas.

Contribuciones de autoría: RLC, RHC, ELC e IGM han participado en la concepción, diseño y redacción del artículo. Además, RHC, CGP, MMM, MCP, NRC, JCH y RLC realizaron la revisión crítica del artículo. RLC realizo el análisis e interpretación de datos y dispuso la asesoría estadística. Todos los autores participaron en la recolección de resultados, el aporte de material de estudio, la obtención de financiamiento y la aprobación de la versión final del artículo.

Fuentes de financiamiento: autofinanciado.

Conflictos de interés: los autores declaran no tener conflictos de interés. 


\section{REFERENCIAS BIBLIOGRÁFICAS}

1. Antón R. Tratamiento endoscópico de la litiasis ureteral mediante láser Holmium en los pacientes del Servicio de Urología del Hospital Nacional Arzobispo Loayza en el período abril 2012 a marzo de 2013 [Tesis Doctoral]. Lima: Facultad de Medicina, Universidad Nacional Mayor de San Marcos; 2014.

2. Thomas K. Epidemiology, Pathogenesis, and Pathophysiology of Urolithiasis. European Urology Supplements. 2010;9(12):802-806. DOI: http://dx. doi.org/10.1016/j.eursup.2010.11.006

3. Vishal R, Jack K. Mechanisms of Stone Formation. Clin Rev Bone Miner Metab. 2011 Dec; 9(3-4):187-197. DOI: $10.1007 / \mathrm{s} 12018-011-9104-8$

4. Herney G, PaolaB,Paola P.Pathophysiology associated with forming urinary stones. Urol Colomb 2016;25(2):118-25 - DOI: 10.1016/j.uroco.2015.12.013

5. Hélö̈s B, Jean-Philippe H. Experimental models of renal calcium stones in rodents. World J Nephrol. 2016 Mar 6; 5(2): 189 194. DOI: 10.5527/wjn.v5.i2.189

6. Fan J, Shen SJ. The role of TammHorsfall mucoprotein in calcium oxalate crystalluria. N-acetylcysteine - A new therapy for calcium oxalate urolithiasis. $\mathrm{Br}$ J Urol. 1994;74(3): 288-293.

7. Grange J, Davey R. Antibacterial properties of propolis (bee glue). J R Soc Med. 1990 Mar; 83(3):159-60.

8. Castaldo S, Capasso F. Propolis, an old remedy used in modern medicine. Fitoterapia. 2002; 73(S1):1-6

9. Soto-Vásquez MR. Metabolitos secundarios, cuantificación de fenoles y flavonoides totales de extractos etanólicos de propóleos de tres localidades del Perú. In Crescendo. Institucional. 2015; 6(2): 22-32.

10. Falcão $S$, Vale N, Gomes P, Domingues M, Freire C, Cardoso S, Vilas M. Phenolic profiling of portuguese propolis by LC-MS spectrometry: Uncommon propolis rich in flavonoid glycosides. Phytochemical Analysis. 2013 Jul-Aug;24(4):309-18. doi: 10.1002/pca.2412.

11. Popova MV, Chinou IB, Marekov IN, Bankova VS. Terpenes with antimicrobial activity from Cretan propolis. J Phytochemist. 2009. Jul;70(10):1262-71. doi: 10.1016/j.phytochem.2009.07.025.

12. Bracho-Pérez JC, Rodríguez-Best C, Llanes F. Triterpenos pentacíclicos en propóleo. Rev Soc Quím Perú. 2009;75(4):439-451.

13. Ingale KG, Thakurdesai PA, Vyawahare NS. Effect of Hygrophila spinosa in ethylene glycol induced nephrolithiasis in rats. Indian J Pharmacol. 2012 Sep-
Oct;44(5):639-42. doi: 10.4103/02537613.100402.

14. Deepika A, Minu S, Suringer S. The Role Of Natural Antioxidants As Potential Therapeutic Agent In Nephrolithiasis. Asian J Pharm Clin Res. 2013;6(3):48-53.

15. Cañas J. Determinación del efecto antinefrolitiásico y diurético de la Manicaria saccifera. [Tesis] Colombia: Facultad de la Salud, Universidad del Valle. 2011.

16. Mejía LM, García-Perdomo HA, Contreras R. Manejo dietario para la prevención de urolitiasis. Urol Colomb. 2014; 23(3):214-218. DOI: 10.1016/ S0120-789X(14)50059-7.

17. Quintero-Mora $M$, Londoño-Orozco A, Hernández-Hernández F, ManzanoGayosso P, López-Martínez R, SotoZárate $\mathrm{C}$ et al. Efecto de extractos de propóleos mexicanos de Apis mellifera sobre el crecimiento in vitro de Candida albicans. Rev Iberoam Micol. 2008 Mar:25(1):22-6.

18. Türkez H, Yousef M, Geyikoglu F. Propolis prevents aluminium damages in rat liver. Food and Chemical Toxicology. 2010 Oct;48(10):2741-6. doi: 10.1016/j. fct.2010.06.049.

19. Koo MH, Park YK. Investigation of Flayonoid Aglycones in Propolis Collected by Two Different Varieties of Bees in the Same Region. Biosci Biotech Biochem. 1997;61(2):367-369

20. Condorhuamán Y. Efecto hipotensor del extracto acuoso de Calceolaria myriophylla Kraenz en ratas hipertensas inducidas por L-NAME [Tesis]. Lima: Cybertesis, Universidad Nacional Mayor de San Marcos; 2009.

21. Lipschitz W, Haddian Z, Kerpscar A Bioassay of diuretics. J Pharm Exp Ther. [Internet] 1943 (citado el 16 de mayo del 2016);79(2):110-116. Disponible en : http://jpet.aspetjournals.org/content $/ 79 / 2 / 97$

22. Li C, Deng L, Sun B. Taurine protected kidney from oxidative injury through mitochondrial-linked pathway in a rat model of nephrolithiasis. Urol Res. 2009 Aug;37(4):211-20. doi: 10.1007/s00240009-0197-1.

23. Khan A, Bashir S, Khan SR, Gilani AH. Antiurolithic activity of Origanum vulgare is mediated through multiple pathways. BMC Complement Altern Med. 2011 Oct 17;11:96. doi: 10.1186/1472-688211-96.

24. Liao L, Richardson KE. The metabolism of oxalate precursors in isolated perfused rat liver. Arch Biochem Biophys. 1972;153(2):438
25. Hosseinzadeh $H$, Khooei AR, Khashayarmanesh Z, Motamed-Shariaty V. Antiurolithiatic Activity of Pinus eldarica Medw. Fruits Aqueous Extract in Rats. Urol J. 2010;7(4):232-237.

26. Vyas B, Vyas R, Joshi S, Santani D. Antiurolithiatic Activity of Whole-Plant Hydroalcoholic Extract of Pergularia daemia in Rats. J Young Pharm. 2011;3(1):36-44. doi: 10.4103/0975-1483.76417

27. Kachchhi N, Parmar R, Tirgar P, Desai T, Bhalodia P. Evaluation of the antiurolithiatic activity of methanolic extract of Celosia argentea roots in rats. Int $\mathrm{J}$ of Phytopharm. 2012; 3(3):249-55.

28. Ghelania H, Chapalaa M, Jadava P. Diuretic and antiurolithiatic activities of an ethanolic extract of Acorus calamus L. rhizome in experimental animal models. J Tradit Complement Med. 2016 Oct;6(4):431436. doi: 10.1016/j.jtcme.2015.12.004

29. Anbu J, Suman S, Kumar KS, kumar RS, Nithya S, Kannadhasan R. Antiurolithiatic Activity of Ethyl Acetate Root Extract of Ichnocarpus frutescens using Ethylene Glycol Induced Method in Rats. J Pharm Sci \& Res. 2011;3(4):1182-1189.

30. Garcia-Nieto VM, Luis-Yanes MI. Litiasis renal. Nefrologia. 2012;7(1):1-6. doi: 10.3265/Nefrologia.2010.pub1.ed80. chapter 1832

31. Righi A, Alves T, Negri G, Marques L, Breyer H, Salatino A. Brazilian red propolis: unreported substances, antioxidant and antimicrobial activities. J Sci Food Agric. 2011 Oct;91(13):2363-70. doi: 10.1002/jsfa.4468.

32. Martinez D, García C, Durang D, Gil J. Caracterización de propóleos provenientes del municipio de Caldas obtenido por dos métodos de recolección. Rev. MVZ Córdoba. 2012;17(1): 2861-2869

33. da Silva RO, Andrade VM, Bullé Rêgo ES, Azevedo Dória GA, Santos Lima BD, da Silva FA, et al. Acute and sub-acute oral toxicity of Brazilian red propolis in rats. J Ethnopharmacol. 2015 Jul; 21(170):6671. doi: 10.1016/j.jep.2015.05.009.

34. Pérez-Pérez M, Sueiro-Oyarzun ML, Boffill-Cárdenas CM, Morón-Rodríguez CFJ, Marrero-Faz CE. Validación de un método in vivo para evaluar la actividad diurética. Rev Cubana Invest Bioméd. 2011;30(3):332-344.

Correspondencia: Rita Karina López Cabanillas Teléfono: 5851708

Dirección: Jr. Puruchuco Mz.12ACLt. 9 Cmte 41 Tablada de Lurin, Villa Maria del Triunfo, Lima. Correo electrónico: ritalopezcabanillas@gmail.com 